\title{
15
}

\section{Security and Management: The Ubiquitous Mix}

\author{
Moderator: Lee LaBARRE, The MITRE Corporation, U.S.A.
}

Standards based management capabilities are becoming widely available in many network and distributed applications products; but unsecured access to the control capabilities they offer could allow accidental or deliberate damage to the network transmission and application services. Also, standards based security capabilities for such products are emerging that will require remote management of their security mechanisms, and security auditing.

The panelists will discuss the concepts relating security and management, the status and relationship of management and security standards, and issues related to their use in the secure management of resources in the data, telecommunications, and client server environments. 